\title{
Structural Analysis of Branched Boron Carbide Nanostructures
}

\author{
B.B. Cao, Z. Guan, and T. Xu
}

Department of Mechanical Engineering and Engineering Science, The University of North Carolina at Charlotte, Charlotte, NC 28223

Boron carbide $\left(\mathrm{B}_{4} \mathrm{C}\right)$ is a very attractive material with extreme hardness, low weight, good neutron absorption, and unique thermoelectric properties, which make it a strong candidate for applications in aerospace, military, nuclear, and microelectronic fields [1]. In recent years, low dimensional $\mathrm{B}_{4} \mathrm{C}$ nanostructures have been synthesized to further enhance their physical or mechanical properties [2]. However, few work has been done upon the detailed analysis of these nanostructures, due to the complicated rhombohedral unit cell $\left(a=b=c=0.516 \mathrm{~nm}, \alpha=\beta=\gamma=65.707^{\circ}\right)$ of $\mathrm{B}_{4} \mathrm{C}$. In this paper, we present a new $\mathrm{B}_{4} \mathrm{C}$ nanostructure with two stages of branches, and discuss its forming mechanism.

The as-grown products were prepared through nickel-assisted co-pyrolysis of diborane $\left(\mathrm{B}_{2} \mathrm{H}_{6}\right)$ and methane $\left(\mathrm{CH}_{4}\right)$ at $1050{ }^{\circ} \mathrm{C}$ for $120 \mathrm{~min}$, pressure controlled at $\sim 40 \mathrm{mTorr}$. The morphology and fine structure were characterized by transmission electron microscopy (JEOL 2100 LaB6 at $200 \mathrm{kV}$ ). At the same time, structural model was built via CrystalMaker ${ }^{\circledR}$.

Fig. 1 shows a typical branched nanostructure with catalytic particles on the ends of nanowires. 0 indicates the root, while $01 / 02$ and $021 / 022$ represent first stage and secondary stage of branch, respectively. High density of stacking faults can be noted for the appearance of modulated contrast along the overall nanostructure. The junction area marked by the black arrow is zoomed in Fig. 2(a). The angle between branch 01 and 02 is measured to be about $107^{\circ}$. High resolution image in Fig. 2(b) confirms large quantity of stacking faults or twins along both 01 and 02 branches. Electron diffraction patterns in region I, II and III, as seen in Fig. 2(c-e), are all on the [001] zone axis, whereas streaking in (c) and (e) is another sign of stacking faults. Thus, the growth direction of branch 01 and 02 can be indexed as normal of (100) and (010) planes accordingly. In the atomic model of $\mathrm{B}_{4} \mathrm{C}$ unit cell (Fig. 3(a)), planes of (100), (010) and (001) are identical. In other words, at the junction area, the growth of atomic layers could happen in either (100) or (010) planes. When viewing along $c$ axis, as shown in Fig. 3(b), interplanar angle between (100) and (010) is $107^{\circ}$, in good agreement with measured angle in Fig. 2(a). In conclusion, the nature of rhombohedral lattice of $\mathrm{B}_{4} \mathrm{C}$ with three identical planes allows the growth of this branched $\mathrm{B}_{4} \mathrm{C}$ nanostructure.

[1] A. K. Suri et al, Int. Mater. Rev. 55 (2010) 4.

[2] Z. Guan et al, J. Mater. Chem. 22 (2012) 9853. 


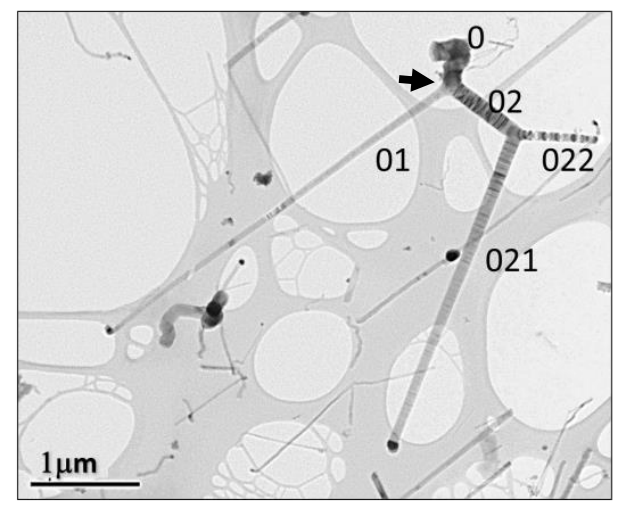

Fig. 1 A typical image of branched $\mathrm{B}_{4} \mathrm{C}$ nanostructure.

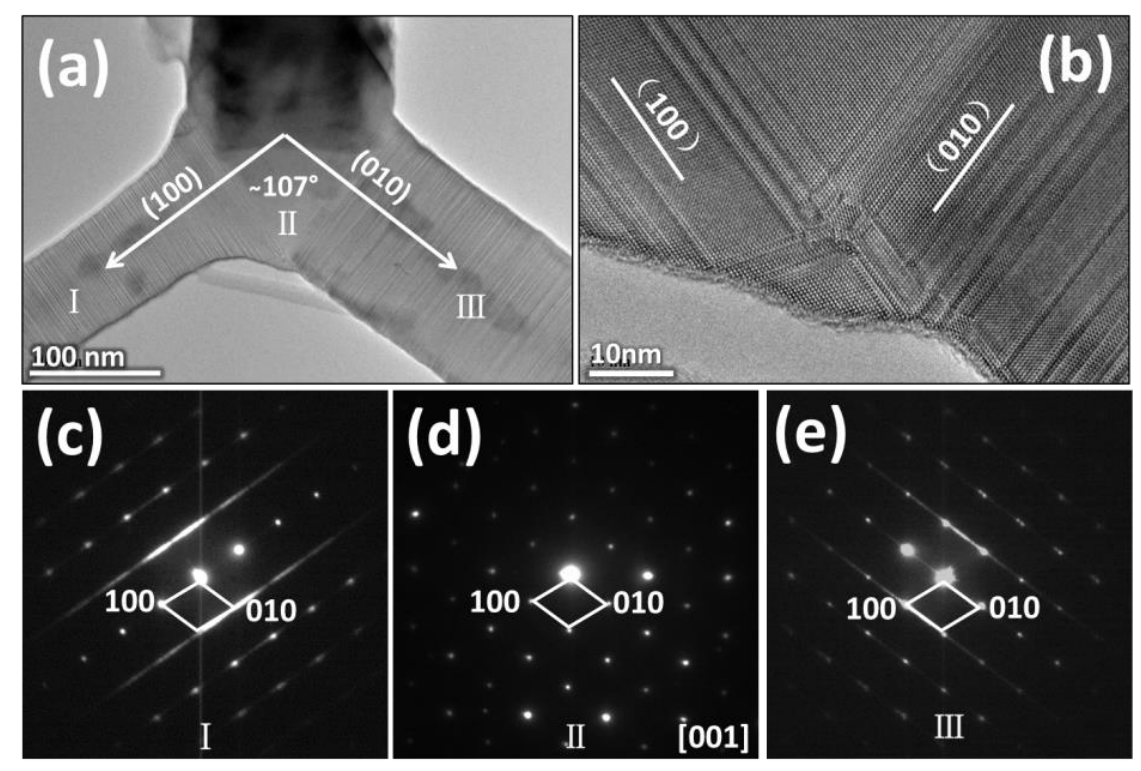

Fig. 2 (a) TEM image of junction area; (b) HRTEM image; (c-e) selected area electron diffraction patterns collected in region I, II, and III in (a).

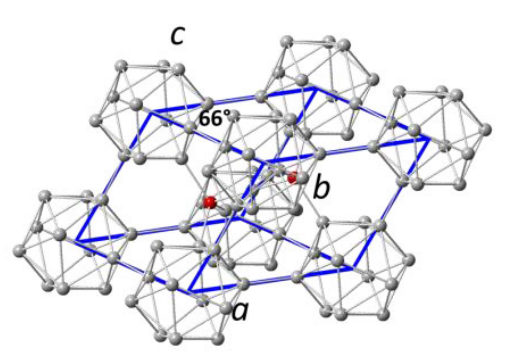

(a)

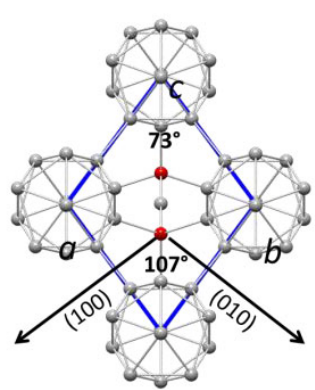

(b)

Fig. 3(a) Rhombohedral unit cell of $\mathrm{B}_{4} \mathrm{C}$; (b) [001] projection. 